\title{
Facteur 4 en Nord-Pas de Calais : impacts socio- économiques
}

Factor four reductions in emissions in the Nord-Pas de Calais region

Anne-Sophie Stevance, Nicolas Houdant et Antoine Bonduelle

\section{(2) OpenEdition}

\section{Journals}

Édition électronique

URL : http://journals.openedition.org/developpementdurable/8846

DOI : 10.4000/developpementdurable.8846

ISSN : 1772-9971

Éditeur

Association DD\&T

Référence électronique

Anne-Sophie Stevance, Nicolas Houdant et Antoine Bonduelle, «Facteur 4 en Nord-Pas de Calais : impacts socio-économiques », Développement durable et territoires [En ligne], Vol. 2, n 1 | Mars 2011 mis en ligne le 31 mars 2011, consulté le 01 mai 2019. URL : http://journals.openedition.org/ developpementdurable/8846 ; DOI : 10.4000/developpementdurable.8846

Ce document a été généré automatiquement le 1 mai 2019.

Développement Durable et Territoires est mis à disposition selon les termes de la licence Creative Commons Attribution - Pas d'Utilisation Commerciale 4.0 International. 


\section{Facteur 4 en Nord-Pas de Calais : impacts socio-économiques}

Factor four reductions in emissions in the Nord-Pas de Calais region

Anne-Sophie Stevance, Nicolas Houdant et Antoine Bonduelle

1 La division par quatre des émissions de gaz à effet de serre à l'horizon 2050 - le « facteur $4 »-$ s'est désormais imposée comme un objectif politique en France, en Europe et dans la majorité des pays développés. Cet objectif découle de la nécessité de stabiliser les températures globales sous les $2^{\circ} \mathrm{C}$, niveau estimé comme dangereux pour les milieux naturels et les sociétés humaines (PNUD, 2007).

2 Ce choix résulte des débats des $O N G$ et de la communauté scientifique, qui ont été diffusés progressivement aux gouvernements. En effet, à $2^{\circ} \mathrm{C}$ de réchauffement et au-delà, des groupes humains voire des continents entiers sont déstabilisés, et des mécanismes irréversibles s'installent. Le coût pour la collectivité mondiale devient incommensurable (Stern, 2006). Les intérêts vitaux des États sont menacés (German Advisory Council on Climate Change, 2008) et il s'agit même pour certains de sauver la civilisation (Brown, 2008).

3 En s'appropriant cet objectif de réduction radicale, les hommes politiques - par exemple le Parlement français en $2005^{1}$ ou le Conseil des Ministres de l'Union Européenne en 2008 (Andersen, 2009) - ont énoncé ou voté un choix majeur. Ceci n'a pas toujours été fait en connaissance de cause : d'une part, ces objectifs issus de travaux scientifiques en pleine évolution pourraient être insuffisants pour limiter la hausse des températures (Hansen, 2008); d'autre part, nos décideurs n'ont pas toujours pris la mesure des implications majeures de ces législations, dans tous les secteurs de la vie économique et sociale.

La décision de division par quatre des émissions de GES n'en reste pas moins une mesure forte. Elle a été suivie par des déclinaisons politiques elles aussi très importantes, comme le « Paquet-Climat-Énergie » européen, ou encore les Plans Climat-Énergie au niveau local afin de se doter d'horizons d'action intermédiaires (à l'échelle du mandat, à 2020). Si un 
cadre politique contraignant est en cours de construction, les conséquences de choix qui s'imposent à tous demeurent incertaines.

5 En effet, cet objectif reste lointain : il défie les horizons temporels de l'action publique et de la décision politique. Il se présente ainsi comme un horizon de ruptures à la fois technologiques, institutionnelles mais aussi économiques et sociétales. Cela pose des interrogations fondamentales en termes de faisabilité technique, de coût, de viabilité économique, d'acceptation sociale, de pertinence environnementale.

Ces difficultés sociales et économiques liées à la mise en œuvre du facteur 4 ont fait l'objet en 2009 d'une recherche prospective impliquant les auteurs du présent article, financée par la Région Nord-Pas-de-Calais. Les travaux présentés ici s'inscrivent dans le cadre d'une étude commanditée par le conseil régional du Nord-Pas-de-Calais portant sur les conditions de mise en œuvre du facteur 4. Ce travail réalisé par les bureaux d'études Énergies Demain et E\&E Consultant vise à identifier les freins et les blocages potentiels (techniques, organisationnels, économiques, sociaux) à l'atteinte du facteur 4.

7 L'étude part d'un territoire - la région Nord-Pas-de-Calais - considéré dans le cadre prospectif de la mise en place de politiques publiques cohérentes avec l'objectif visé de division des émissions. Une fois ce cadre posé, l'étude interroge les effets socioéconomiques des politiques de facteur 4 sur le territoire, les activités et les populations. Le cadre prospectif est construit sur la base des scénarios nationaux et régionaux de division par quatre des émissions existantes. En particulier, on a identifié une série de chantiers incontournables sur lesquels l'action publique devra porter, et qui représentent l'essentiel des potentiels de réduction d'émissions dans le périmètre régional :

- Le ralentissement et/ou arrêt de l'étalement urbain ;

- Les infrastructures de transport ;

-L'amélioration des véhicules ;

- La substitution des modes de transport de passagers;

- La substitution des modes de transport pour le fret;

- Les politiques spécifiques sur la sidérurgie et les ciments ;

- L'efficacité carbone dans les entreprises ;

- La sobriété et l'efficacité dans les équipements ménagers et de bureau ;

- La rénovation et la réhabilitation des logements et des bureaux;

- L'extension des réseaux de chaleur ;

La transformation du réseau électrique ;

- L'agriculture et la sylviculture.

8 Par ailleurs, les émissions amont comme celles liées à la consommation alimentaire des ménages et à leurs émissions indirectes comme celles du tourisme aérien, de même que les gaz industriels et les émissions importées sous forme de demi-produits sont considérés séparément.

9 L'étude s'intéresse ainsi d'abord aux émissions directes sur lesquelles les acteurs régionaux peuvent agir, ou ont tout au moins des capacités d'influence. Les émissions amont renvoient, elles, à une réflexion qui dépasse les périmètres de l'étude et qui nécessite de développer l'analyse sur une base non plus territoriale mais sectorielle : des études de filière précises seront nécessaires pour identifier les circuits d'échanges internationaux, répartir les "responsabilités » liées aux émissions et définir des périmètres de quantification cohérents. On notera tout d'abord que certains des chantiers, comme celui de l'alimentation, ont dû être abordés par une approche qualitative, en attendant des travaux plus détaillés. Le cas du Nord-Pas de Calais, à la 
différence d'une agglomération prise isolément ou d'une zone à dominante tertiaire, est celui d'une région fortement industrielle dont la production de biens matériels (aciers, automobiles, verrerie, agro-alimentaire...) reste conséquente. Cela nous nous permet d'estimer qu'une approche par les émissions directes seules est cohérente et ne sousestime pas de façon importante le bilan régional des émissions de gaz à effet de serre. Par ailleurs, les exemples développés plus loin de rénovation de l'habitat et d'évolution des transports de passagers, renvoient à des bilans avant tout directs. Ces chantiers utilisent des matériaux de construction ou des véhicules produits en région ou au moins sur notre continent. De plus, l'incidence des émissions liées à la fabrication des véhicules demeure faible vis-à-vis de leur consommation directe.

10 Enfin, les accords internationaux et les documents-cadres (autrement désignés par " régime climatique ») qui définissent l'intervention des États en matière d'atténuation reposent sur les émissions directes déclarées par ces États. Via l'État et l'Union Européenne, le régime en vigueur est celui du Protocole de Kyoto, de la Loi d'Orientation sur l'Énergie du 13 juillet 2005 et du Paquet Climat Énergie. Ces textes prennent pour base les émissions directes. L'objectif n'était pas, dans le cadre de cette étude, de discuter de l'opportunité du facteur $4 \mathrm{ni}$ de son cadre politique de décision, mais de tenter d'en appréhender les conséquences sociales et économiques sur un territoire donné.

11 La description du contenu possible des politiques incontournables du facteur 4 a déjà été amorcée, en particulier dans les scénarios conduits au niveau national (de Boissieu, 2006 ; Radanne, 2004) et régional (Virage Énergie Nord-Pas-de-Calais, 2007). Initialement, la politique climatique est appréhendée comme un horizon lointain. Pour être utile au politique, le scénario devra découper cet objectif en politiques-cadres assorties de programmes d'action et d'échéances de mise en œuvre et par là, alimenter des débats pour définir les choix. Pour chacune de ces actions, il s'agit d'identifier les différents obstacles: difficultés politiques, catégories sociales impactées, et ceci au regard des spécificités des territoires, de la structure des activités et de leurs contraintes.

\section{Le cadre de l'analyse}

\subsection{L'appropriation de l'objectif du facteur 4 , un enjeu politique et social en soi}

12 L'objectif des analyses menées dans l'étude est de comprendre les conditions de faisabilité du facteur 4. De ce point de vue, cette prospective est à la fois technique mais aussi politique. Elle vise à engager une discussion en vue de l'élaboration d'une stratégie informée de réduction drastique des émissions de GES, tout en cherchant à minimiser les effets négatifs pour les citoyens et pour la collectivité.

Le dispositif d'analyse proposé doit permettre d'identifier, de façon la plus rigoureuse et la plus exhaustive possible, les champs de contrainte et les conditions pour une prise en charge par les acteurs d'un objectif encore peu opérationnel. La réalisation du facteur 4 nécessitera des innovations politiques et sociales, et pas seulement des changements réglementaires et technologiques. Enfin, le cadre d'analyse doit nous permettre de tenir compte de la dynamique des territoires et des capacités d'action des acteurs régionaux. Il doit aussi rester suffisamment souple pour ne pas imposer des solutions. 

régional ainsi que des réglementations adoptées ou en cours de discussion comme la loi Grenelle II. Un travail de modélisation a été réalisé pour les secteurs les plus émetteurs afin de comprendre les cadres proposés dans les scénarios de référence, pour en déduire les mesures nécessaires et apprécier les dispositifs complémentaires à mettre en place.

\subsection{La modélisation comme révélateur}

Le recours à la modélisation technique vise à analyser et imaginer les futurs possibles (anticipation des changements prévisibles ou potentiels) et à proposer des déclinaisons imagées des objectifs sectoriels du facteur 4 : modéliser, c'est tenter de traduire ce que pourrait être la contrainte énergétique associée à cet objectif. Par exemple, la diminution forte des émissions dans le secteur résidentiel régional correspond à un rythme de réhabilitation annuel des logements qui peut être irréaliste. Autre exemple, les objectifs en matière de développement $\mathrm{du}$ transport en commun impliqueraient que l'agglomération de Lille approche vers 2050 une densité et un niveau de service similaire à celle de Paris et de sa petite couronne. La prospective, qui reste avant tout un exercice exploratoire, permet de comprendre et de mesurer mais implique de faire des paris sur l'avenir. Elle part de la définition rigoureuse d'hypothèses développées au regard du passé récent, afin d'extrapoler des tendances lourdes : augmentation du prix des énergies fossiles et de l'électricité, mise en œuvre d'un principe de taxation carbone.

Ce premier dispositif d'analyse des scénarios ambitionne de «faire parler le monde muet» (Latour, 1999: 28) - à savoir les émissions de gaz à effet de serre, les consommations d'énergie - de représenter pour donner à voir et ainsi, de rendre tangibles des objectifs globaux. La simulation prospective n'est pas qu'une construction théorique, car elle permet de se projeter sur des horizons temporels difficilement saisissables et d'appréhender des objets complexes. L'exercice réalisé répond également à une nécessité d'agir et, en ce sens, se doit d'être «utile» pour la décision et l'action publique. Le dispositif prospectif déployé ici est donc, d'une part, un outil de description des conséquences économiques et sociales du facteur 4 pour les ménages du Nord-Pas-deCalais. D'autre part, c'est un outil d'interrogation de la faisabilité des objectifs facteur 4 qui permet de mesurer l'ampleur des politiques nécessaires et leur réalisme.

S'efforçant de manipuler des ensembles directement appréhendables par des nontechniciens, les travaux décrits visent à élargir la réflexion pour débattre du souhaitable, du faisable et de l'acceptable en termes d'action publique, de mobilisation des acteurs, de changements de comportements, domaine encore lacunaire. En effet, la complexité des problématiques posées par la réponse au changement climatique nécessite de les poser collectivement et en quelque sorte de « socialiser les connaissances» (De Boissieu, 2006 : 31). Les scénarios amorcent la construction d'un récit collectif contribuant à l'élaboration de politiques publiques de réduction des émissions de GES. En tenant compte des contraintes opérationnelles, le cadre d'analyse a pour ambition de contribuer à établir une « communauté de référence » (Lecœur, $2008: 230-235)$ sur les objectifs envisageables, les problèmes de faisabilité technique, économique et sociale, les zones d'incertitude, les secteurs et les populations vulnérables dans la région Nord-Pas-de-Calais. 


\section{Les enjeux sociaux et économiques de la mise en œuvre du facteur 4}

18 La mise en œuvre du facteur 4 suppose une diminution drastique des émissions de gaz à effet de serre et par conséquent des consommations d'énergie. Cette contrainte énergétique impactera les territoires, les activités et les populations de façon différenciée: elle implique des modifications en profondeur de la physionomie des territoires, des modes de vie, de déplacement, de production et de consommation. Deux chantiers «incontournables", la réhabilitation de l'habitat ancien et la mobilité individuelle ont été choisis en particulier. Ces ensembles concentrent en effet les facteurs de mise en difficulté des ménages. Ils sont de plus urgents et centraux à la fois en termes quantitatifs et en termes de consensus nécessaires (Radanne, 2005).

\subsection{Logement}

\subsubsection{La contrainte énergétique, facteur de vulnérabilité des ménages}

19 Tout d'abord, il convient de définir le niveau de la contrainte liée au coût des énergies carbonées qui pèsera sur les ménages. La prospective sur les prix de l'énergie est une matière difficile, mais les valeurs encadrantes de la plupart des études convergent désormais sur une augmentation des prix de l'énergie et du coût des émissions. En effet, il est probable que la production pétrolière atteigne ses limites dans un horizon proche. Un tel avenir est décrit à la fois par les partisans des théories du " pic pétrolier » (Deffeyes, 2001) qui estiment que la production conventionnelle d'hydrocarbures est déjà en train de décliner (Criqui, 2006). Cette vision a été rejointe pour le moyen terme par les prospectivistes de l'Agence Internationale de l'Énergie (AIE), une organisation intergouvernementale dont les analyses sont «visées » par les gouvernements des pays membres et qui ne peut être soupçonnée d'alarmisme (AIE, 2009).

Or, la part du budget consacrée aux énergies est un facteur de vulnérabilité important pour les ménages (ADEME, 2008). On entend ici la probabilité de voir se détériorer sa situation ou ses conditions de vie. Cette vulnérabilité varie selon la composition et l'âge du ménage, le niveau de revenu et le lieu de résidence. Ainsi, la part du prix du pétrole brut dans le budget des ménages (de l'ordre de $4,4 \%$ en moyenne) passe à $4,9 \%$ du budget total pour les $10 \%$ de la population les moins fortunés, et à $4 \%$ pour les $10 \%$ les plus aisés. On observe des variations du même ordre en fonction du lieu d'habitation (de 3,9 \% en Île de France à 5,0 \% en milieu rural) et de l'âge du ménage (de $4 \%$ pour les $25-35$ ans, à $5 \%$ pour les plus de 75 ans) (Kasparian, 2008).

\subsubsection{Des ménages déjà soumis à la contrainte énergétique dans le Nord-Pas-de- Calais}

21 Avant d'engager la prospective, un examen de la situation présente est nécessaire. La carte ci-dessous représente la part de l'énergie liée au logement dans le budget des ménages aujourd'hui. 
Carte $\mathrm{n}^{\circ} 1$. Situation actuelle - Part du budget des ménages consacré aux énergies liées au logement dans le revenu fiscal médian

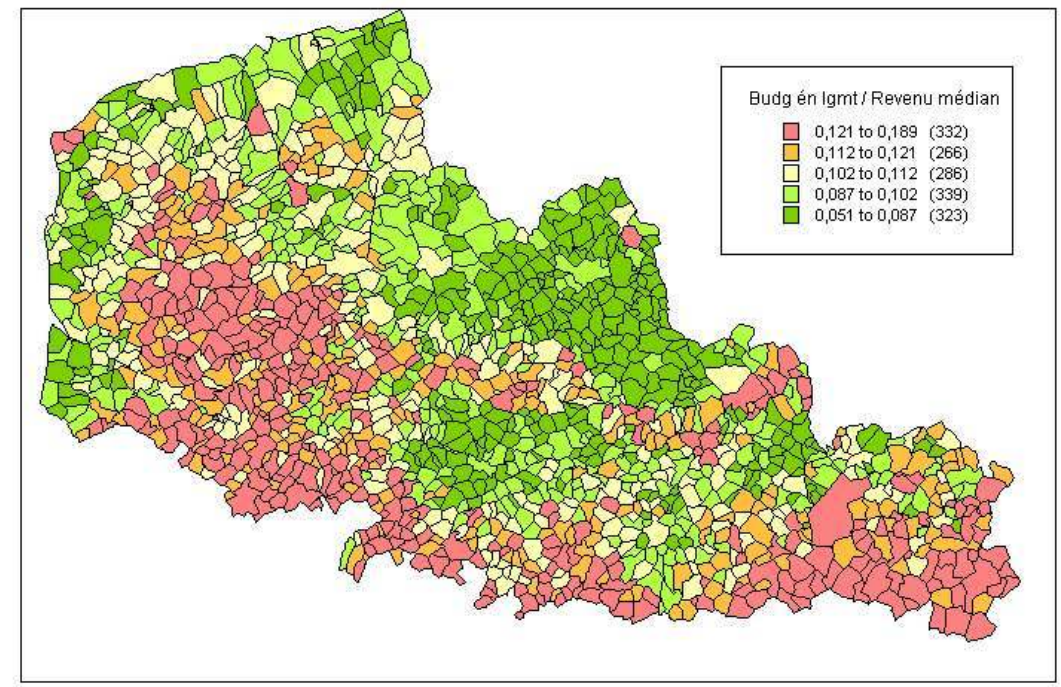

Source : Énergies Demain, $2007^{2}$

consacré aux énergies consommées pour le logement est plus importante : espaces ruraux (Sambre-Avesnois, Artois Cambrésis, Audomarois-Ternois-Sept Vallées) et agglomérations (bassin minier de Béthune à Valenciennes). Plusieurs facteurs expliquent ces contrastes, et en premier lieu les revenus des ménages. Les zones mises en évidence sur la carte $\mathrm{n}^{\circ} 1$ (ci-dessus) correspondent aux ménages dont le revenu fiscal médian est le plus bas ${ }^{3}$. La consommation dépend, elle, de la qualité et de la taille des logements. Ainsi, les populations peu fortunées, habitant dans des logements individuels vétustes ou de grande taille connaissent d'ores et déjà des difficultés. Cette réalité peut être aggravée par la source d'énergie du chauffage, notamment fioul et électricité. De plus, la part des habitants âgés de plus de 65 ans dans ces territoires est plus élevée qu'ailleurs ${ }^{4}$ : cela constitue un facteur de vulnérabilité potentielle car les personnes âgées passent en moyenne plus de temps dans leur logement que la population active et sont aussi plus exposées aux conséquences sanitaires du sous-chauffage (Boardman, 2005). Elles rencontrent aussi des difficultés d'accès au crédit ou sont moins orientées vers des stratégies patrimoniales d'investissement pour réhabiliter leur logement. Cependant, le critère du revenu domine.

Sans aucune intervention majeure sur les logements, la part du budget énergie lié au logement passera de $2350 €$ (soit en moyenne $9 \%$ du budget total des ménages) aujourd'hui à $7400 €$ en 2050 (soit $29 \%$ du budget total) du fait de l'augmentation du prix des énergies. La carte $\mathrm{n}^{\circ} 2$ ci-dessous montre cette dégradation des conditions de vie des ménages en cas de stagnation du pouvoir d'achat, et en raisonnant à monnaie constante. On observe que les fourchettes hautes (c'est-à-dire la part du budget énergie logement comprise entre $11 \%$ et $19 \%$ du budget total des ménages, et au-delà) représentées en rose et orange ci-dessus et qui correspondaient aux situations déjà décrites comme vulnérables deviennent très répandues voire dominantes en 2050 . 
Carte $n^{\circ} 2$. Part du budget consacré aux énergies logement dans le revenu fiscal médian, par ménage en 2050

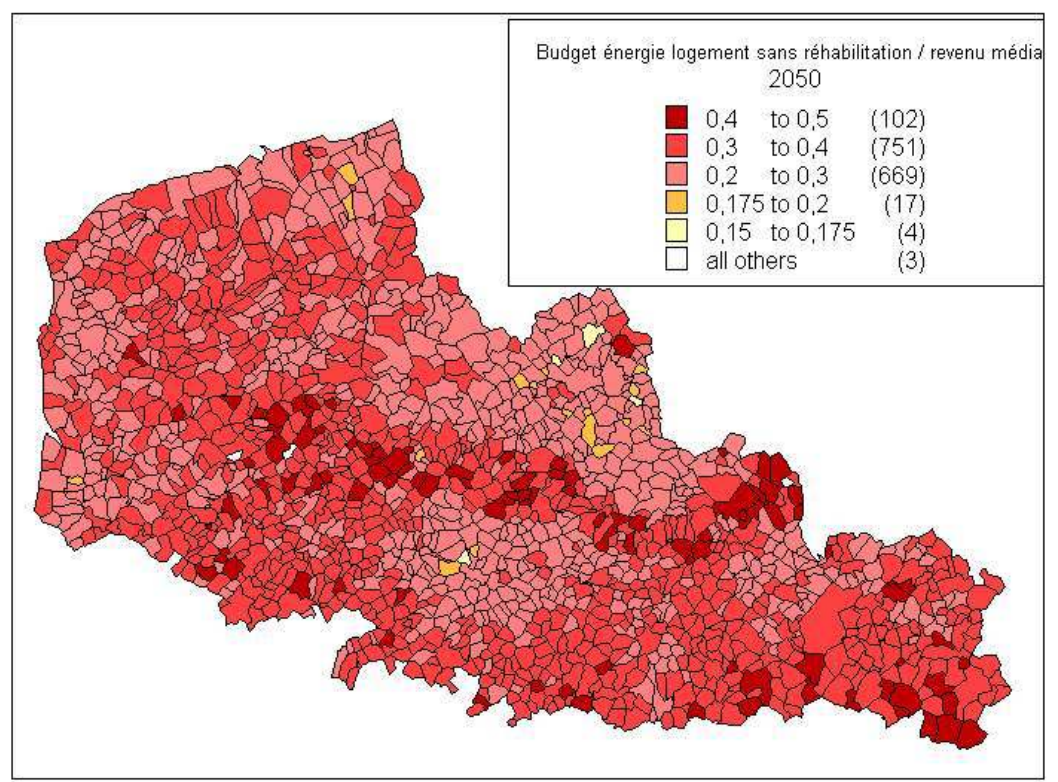

Source : Énergies Demain, 2007 de leur logement dans le cadre d'un prêt type "taux zéro", afin de diminuer les consommations et ainsi, compenser, au moins en partie, la hausse du coût des énergies. Le graphique ci-dessous modélise plusieurs situations-type :

- Le ménage ne réhabilite pas son logement (courbe bleue);

- Le ménage réhabilite son logement en 2015 (courbe rouge);

- Le ménage réhabilite son logement en 2030 (courbe verte). 
Graphique $n^{\circ} 1$ : Annuités du coût des énergies et travaux de réhabilitation liés au logement, 2007-2050

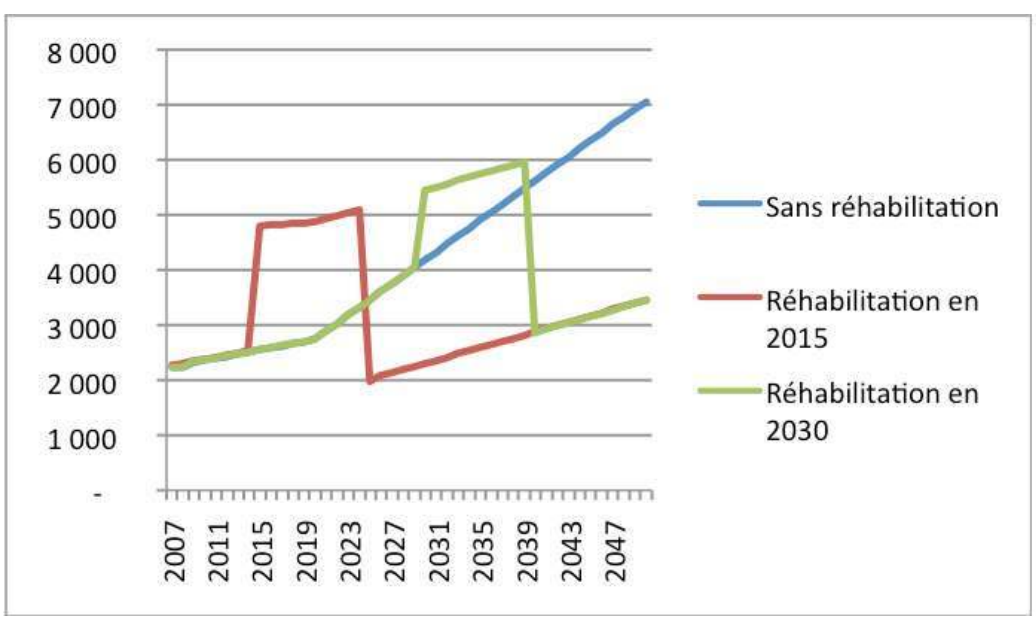

SOURCE : ÉNERgIES DEMAIN, 2007

La courbe bleue augmente proportionnellement au coût des énergies. La répartition actuelle des énergies consommées est alors conservée. La brusque augmentation d'annuités en 2015 (courbe rouge) ou 2030 (courbe verte) correspond au début du remboursement du prêt pour les travaux de réhabilitation. Ce surcoût disparaît dix ans plus tard, à la fin du remboursement des travaux. La modélisation se base avant tout sur l'hypothèse du financement des travaux par l'éco prêt à taux zéro (PTZ), considérée ici comme pérenne. Il s'agit en effet d'une mesure phare gouvernementale censée porter l'effort de lutte contre le changement climatique dans le secteur résidentiel. Les prêts bonifiés semblent en effet plus pérennes que les crédits d'impôt. Ces derniers doivent prendre fin en 2012 et sont déjà moins pris en charge par l'État, avec par exemple une baisse des taux de ces crédits de $25 \%$ à $15 \%$ sur l'investissement dans des parois vitrées à partir de 2010. D'autres mesures comme les tarifications progressives peuvent avoir un impact social différencié pour un coût global similaire, mais de telles mesures ne sont pas actuellement employées (Saglio, 2010).

Il faut souligner que l'augmentation du coût des énergies ne sera compensée que partiellement par les économies d'énergie liées aux travaux de réhabilitation. De plus, nous avons ici modélisé les économies potentielles qui pourraient être réalisées par une intervention sur le bâti. Il convient de prendre également en compte la dimension sociale de la consommation d'énergie: ainsi, de nombreux facteurs sociaux liés aux comportements d'usage des logements "perturberont» la réalisation des objectifs du facteur 4. En particulier, les efforts consentis pour la réhabilitation du logement peuvent se traduire partiellement par une recherche de confort supplémentaire et ainsi diminuer les économies d'énergie potentielles liées à l'amélioration de la qualité thermique du bâti. À ce stade, une discussion importante est celle des effets rebond. Une moindre dépense énergétique diminue la pression économique sur les ménages. Dans certains cas des ménages modestes se chaufferont mieux. Au-delà, on s'interroge sur les effets du relâchement de la contrainte budgétaire obtenu grâce à l'isolation des logements. Cependant, les effets rebonds observés jusqu'à maintenant s'inscrivent dans un contexte de relative abondance des énergies fossiles et de prix bon marché. Le phénomène pourrait être encore réduit par l'augmentation forte des prix des énergies (ADEME, 2010). 
Cependant, le maintien de niveaux de prix élevés pour l'énergie nous laisse présager, malgré les travaux de réhabilitation, la persistance de situations de précarité énergétique chez les ménages.

\subsubsection{Le facteur 4, révélateur d'enjeux économiques et sociaux} vulnérabilité majeurs. On peut souligner par exemple que le prêt à taux zéro qui constituera le dispositif financier principal dans les prochaines années pour inciter les ménages à la réhabilitation de leur logement, vise avant tout les ménages solvables et les propriétaires occupants. Ce type de dispositif ne concerne pas les ménages qui interviennent sur leur logement en auto-réhabilitation. Les ménages ne disposant pas des capacités de financement suffisantes (tels que les ménages précaires ou endettés) ainsi que les locataires seront aussi plus vulnérables. Le renchérissement du coût des énergies risque alors de toucher d'autant plus durement ces catégories. De plus, d'après notre analyse, les travaux de réhabilitation présentent des temps de retour sur l'investissement longs, en l'état des dispositifs financiers existants, ce qui peut présenter un obstacle majeur pour les ménages en situation de mobilité géographique ou bien les personnes âgées.

31 La vulnérabilité est multidimensionnelle et ne peut se résumer aux seules capacités de financement. Les comportements d'usage et les ressources (économiques, sociales, cognitives) dont dispose l'usager sont des facteurs importants à prendre en compte afin d'identifier les situations de vulnérabilité et les dispositifs à mettre en place: la connaissance des dispositifs les plus performants, la propension sociale à investir dans des travaux ou des achats d'équipements (d'où une perception de l'investissement dans l'isolation de la maison comme peu rentable économiquement et socialement), la recherche des informations, les capacités à négocier (avec le propriétaire bailleur, le syndicat de copropriété etc.) influent sur la capacité des ménages à réduire leurs consommations d'énergie. Ainsi, les « occupants captifs » tels que les personnes âgées, les personnes invalides, les populations à très faible revenu ne disposent pas nécessairement des ressources financières, relationnelles et sociales suffisantes pour engager un projet de réhabilitation (Cérézuelle, $2007: 10$ ).

Si les situations sociales et financières sont diverses, il en va de même pour les pratiques de réhabilitation. On peut notamment prendre l'exemple de l'autoréhabilitation, répandue dans le Nord-Pas-de-Calais: une étude menée par le CERPEAU à la fin des années 1980 à Denain souligne l'importance de ces pratiques. Les pratiques de l'autoréhabilitation ou d'autoconstruction sont historiquement importantes dans les milieux populaires. Cette "production "sociale" du logement» valorise un capital technique et social du ménage et présente des enjeux importants en termes d'appropriation du logement. De plus, ces pratiques s'ancrent dans des réseaux sociaux informels. La diffusion des niveaux de performance énergétique nécessaires pour atteindre le facteur 4 nécessite de prendre en compte la diversité des pratiques sociales de réhabilitation que l'approche économique ne met que partiellement à jour. qualitatifs et quantitatifs qu'il faudra opérer. L'amélioration qualitative des logements renvoie à leurs qualités thermiques (isolation, systèmes de chauffage, etc.) conformément aux réglementations thermiques. Mais surtout, l'habitat montre une inertie forte en 
raison de la durée de vie moyenne des logements contrairement à d'autres équipements tels que les véhicules ou encore les appareils électroménagers. Cela souligne les difficultés pour modifier profondément la physionomie du parc en l'espace de 30 à 40 ans. À raison d'un taux de démolition de 50000 logements par an - ce qui est bien supérieur au taux annuel estimé actuellement d'environ 30000 logements - il faudrait près de 500 ans pour détruire et renouveler en totalité le parc existant en 2000 (Traisnel, 2001 : 14). Poser la difficulté en ces termes permet d'appréhender le défi politique et social du facteur 4 et les limites des instruments politiques existants.

Le parc de logements a connu un rythme de croissance important durant les Trente Glorieuses : le nombre de mises en chantier atteint 550000 logements par an au début des années 1970 (INSEE, 1996). Il s'établit à moins de 300000 au terme de la décennie passée. Rétrospectivement, nous pouvons constater qu'un effort important dans le domaine du bâtiment peut être consenti par nos sociétés. Néanmoins, des questions se posent en termes de dispositifs à mettre en place à destination des ménages, du rôle des collectivités et de l'État dans la planification territoriale de la réhabilitation des logements voire de la destruction-reconstruction des logements les plus vétustes.

Une politique de réhabilitation de cette envergure aura des conséquences économiques et sociales importantes, au-delà des interrogations concernant la capacité de financement des ménages décrit plus haut. Elle implique des efforts massifs en termes de formation des artisans et des professionnels du bâtiment aux techniques de l'éco-construction et à l'utilisation des matériaux performants. Une telle politique suppose également des reconversions importantes vers ces secteurs déjà confrontés à des difficultés de recrutement. Enfin, les modalités de vérification de l'application de la réglementation thermique conditionnent également le succès de ce chantier de grande ampleur.

\subsection{Aménagement du territoire et déplacement}

La hausse du prix des énergies aura également des conséquences économiques et sociales majeures dans le domaine du transport. La mobilité des personnes est aujourd'hui marquée par le "système de l'automobile ", selon l'expression de Frédéric Héran : non seulement le réseau de transport et de services est organisé autour de l'automobile mais il conditionne aussi l'accessibilité des territoires et des emplois (Héran, 2006). La remise en question de cette relation peut être explosive socialement : c'est ainsi que les victimes de l'ouragan Katrina à la Nouvelle-Orléans étaient souvent des personnes ne disposant pas d'automobile qui n'ont pas pu fuir l'inondation; cette ségrégation par le transport s'est cumulée avec la pauvreté pour aboutir à une situation sociale explosive (Cannovao, 2008). Si la relation entre la densité urbaine et les systèmes de transport est complexe, la généralisation de la voiture a accompagné l'urbanisme extensif : en effet, l'usage de la voiture croît avec l'éloignement du centre (Traisnel, 2007).

Dans le cadre de notre analyse, l'aménagement du territoire et le système de transport associé constituent un axe de réflexion majeur car ils sont respectivement le support et les vecteurs de l'ensemble des activités économiques et sociales. La modélisation a porté à la fois sur l'amélioration des technologies de déplacement et sur les possibilités d'influer sur les choix modaux notamment par le biais des structures urbaines. 


\subsubsection{L'augmentation du coût des énergies remet en cause les pratiques de mobilité actuelles}

Il convient tout d'abord de faire un état des lieux de la part du transport dans le budget des ménages du Nord-Pas de Calais.

Carte $n^{\circ}$ 3. Budget consacré au transport, par ménage - Situation actuelle

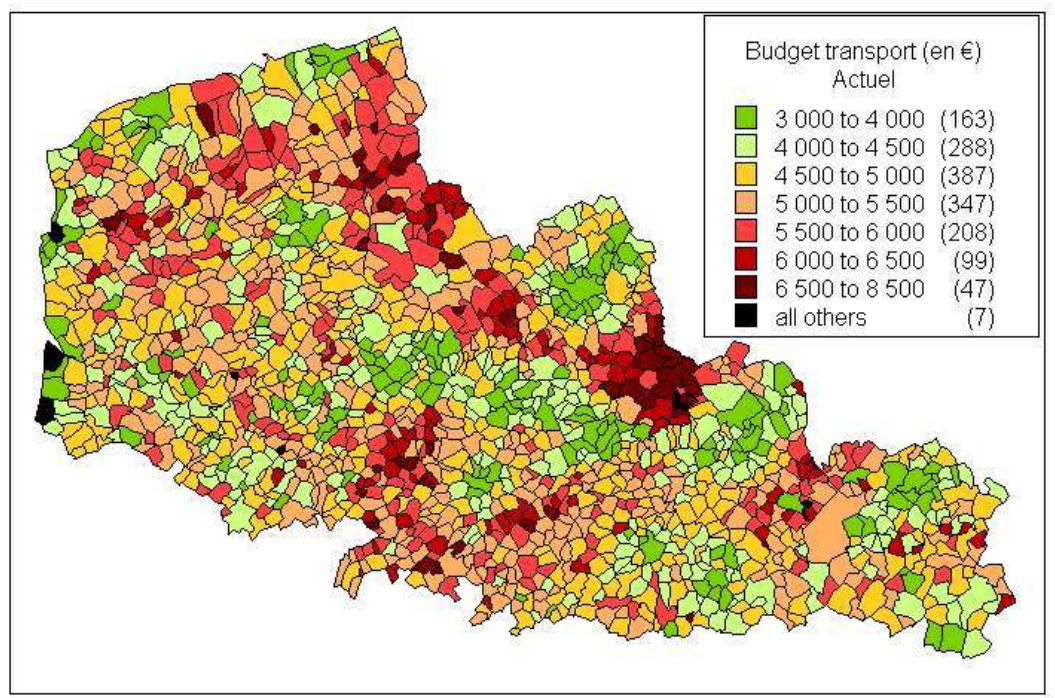

SOURCE : ÉNERgIES DEMAIN, 2007

Là encore, la carte permet de mettre en évidence les contrastes entre territoires: les ménages des zones périurbaines (notamment autour d'Arras et de Lille, et dans une moindre mesure de Valenciennes) consacrent un budget important au transport. Ceci est dû à des distances parcourues élevées par ménage $\left(25200 \mathrm{~km}^{6}\right.$ par an en zone périurbaine contre 20400 en moyenne dans la Région) et à une part légèrement plus importante de la voiture ( $62 \%$ des $\mathrm{km}$ parcourus contre $59 \%$ en moyenne). On peut également souligner que les ménages résidant dans la zone frontalière au nord-ouest de l'aire métropolitaine de Lille engagent des dépenses de transport également importantes. Ce point peut s'expliquer par des mouvements pendulaires transfrontaliers ou l'influence de la bipolarisation Lille-Dunkerque. Ce sont finalement les ménages habitant en ville (communauté urbaine de Lille, Valenciennes, le bassin minier, Cambrai, Arras, Boulogne, Dunkerque) qui ont le budget transport le plus faible. Ces territoires disposent d'une desserte en transports en commun plus importante que le reste du territoire régional.

L'exercice de simulation à 2050 - à distances parcourues inchangées - permet d'évaluer l'effort budgétaire que les ménages devront consentir. La carte ci-dessous montre que même les ménages sans difficultés en 2007 et disposant de conditions favorables (centres urbains disposant d'alternatives à la voiture) se retrouveront en situation plus difficile face à la flambée du prix des carburants. 
Carte $n^{\circ} 4$ Budget consacré au transport, par ménage en 2050

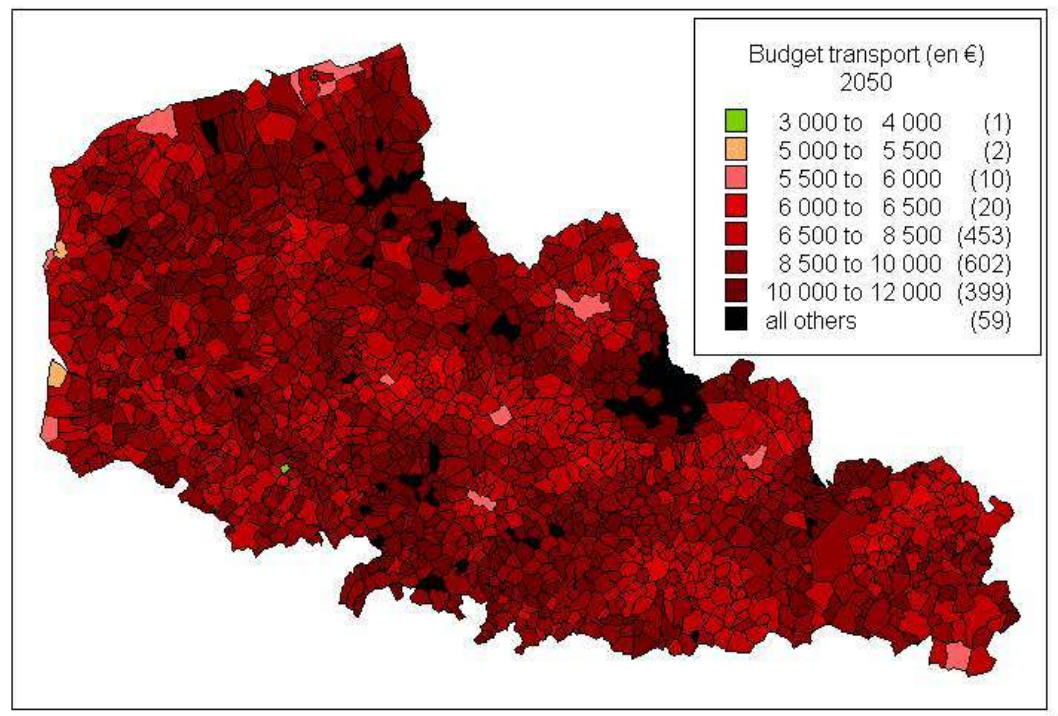

SOURCE : ÉNERgIES DEMAIN, 2007

41 Sans intervention pour diminuer les distances parcourues et substituer les transports collectifs à la voiture particulière, le budget consacré au transport en 2050 grimpe à $30 \%$ du revenu disponible médian des ménages ; il est de $16 \%$ aujourd'hui avec des contrastes importants entre pôle urbain et couronne périurbaine d'une part et pôle urbain zones rurales d'autre part. On passerait de $4100 €$ par an en moyenne aujourd'hui à $4700 €$ en 2020 et à $7700 €$ en 2050. De plus, l'augmentation des dépenses liées au transport ne touchera pas uniformément les populations : elle pourrait renforcer la vulnérabilité des territoires comptant déjà des situations sociales défavorables (Sambre-Avesnois, Audomarois-Ternois-Sept Vallées) et les banlieues des principales agglomérations.

\subsubsection{Quelles évolutions de la mobilité ?}

Différents leviers pourront être actionnés pour atteindre une diminution par quatre des émissions liées à la mobilité des personnes. Trois leviers ont été examinés dans le cadre de notre simulation prospective, en se basant sur la description des trajets domiciletravail décrite par l'INSEE :

- Le covoiturage revient à augmenter le taux d'occupation de la voiture. C'est un levier particulièrement intéressant pour les territoires ruraux pour lesquels il est difficile de développer une offre de transport en commun rentable. Le taux d'occupation de la voiture est aujourd'hui de 1,1 personne par déplacement. Dans l'exercice, nous avons poussé cette part à 1 trajet sur 2 en co-voiturage pour les trajets domicile-travail ;

- Le levier technologique est prépondérant dans les scénarios facteur 4, parce que la durée de vie d'un véhicule - 15 ans en moyenne - permet d'envisager un renouvellement complet du parc. Dans la continuité des normes envisagées dans le Grenelle, l'exercice prend en compte les hypothèses suivantes : apparition des $<120 \mathrm{~g} \mathrm{CO}_{2} / 100 \mathrm{~km}$ à partir de 2008, puis des $<85 \mathrm{~g}$ à partir de 2015, et des $<60 \mathrm{~g}$ à partir de 2026 ainsi qu'une pénétration massive de l'électricité et du gaz naturel pour les véhicules (GNV) pour les transports collectifs;

- Le report vers des modes de transport alternatifs à la voiture particulière : c'est le levier 
le plus ambitieux car il implique une intervention sur le système de transport et, de fait, sur la structure des territoires. Il s'agit notamment d'un report modal vers les transports en commun pour les trajets de longue distance et vers les modes doux (marche et vélo) pour les trajets de courte distance. L'intervention sur les choix modaux suppose une évolution des pratiques de mobilité. Le diagramme ci-dessous présente la distribution des parts modales sur un territoire de centre urbain pour parvenir à une situation compatible avec le facteur 4 :

\section{Graphique 2. Simulation des transferts de parts modales - Zone centrale - Parts modales en fonction de la distance}

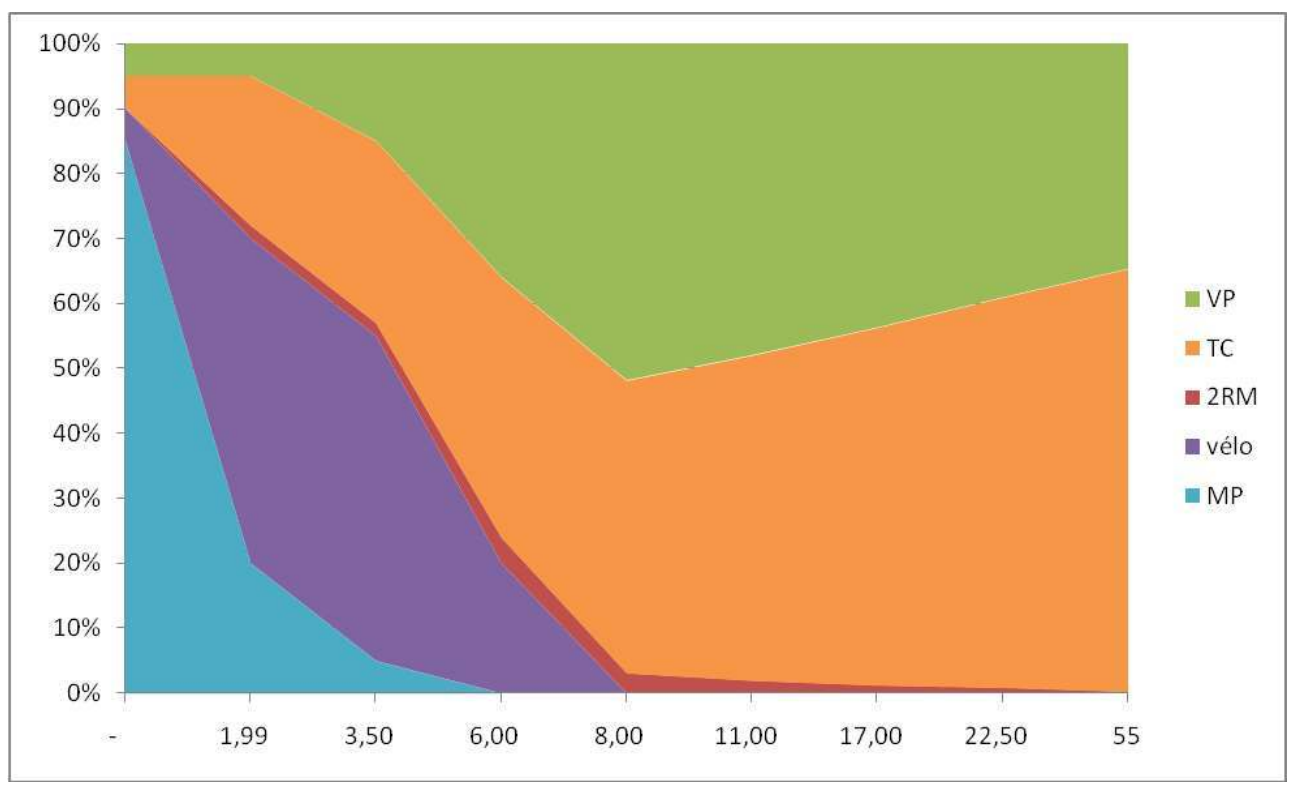

SOURCE : ÉNERgIES DEMAIN, 2007

Les transports en commun en longue distance dans ce scénario représentent $65 \%$ des déplacements, soit une part beaucoup plus importante que celle observée aujourd'hui en Île-de-France : +25 points de parts modales, multiplication par deux de la part observée en commune centre de l'agglomération lilloise, valenciennoise, douaisienne ou béthunoise. La forte utilisation du vélo renvoie à des hypothèses également très fortes en termes de report modal, basées sur la situation actuelle dans la ville de Copenhague. Pour l'ensemble des déplacements entre 0 et $5 \mathrm{~km}, 50 \%$ des déplacements sont effectués en vélo ( 1 à $3 \%$ actuellement). Cette part passe à $20 \%$ pour les trajets entre 5 et $7,5 \mathrm{~km}(0,1 \%$ actuellement) et devient par contre quasi-nulle au-delà de $8 \mathrm{~km}$.

Pour atteindre le facteur 4, ces différents leviers n'interviennent pas à parité : le facteur technologique permet de réaliser à lui seul un facteur 3. Le facteur 4 implique une combinaison du levier aménagement du territoire, covoiturage et amélioration de la performance énergétique des véhicules. Le tableau ci-dessous permet de donner des ordres de grandeur de la portée de chaque levier.

Tableau $\mathrm{n}^{\circ} 1$. Évolution des émissions de gaz à effet de serre

\begin{tabular}{|l|l|l|}
\hline En millions de km. Voyageurs & $\begin{array}{l}\text { Émissions de gaz à effet de serre } \\
\text { (en teq CO2) }\end{array}$ & $\begin{array}{l}\text { Écart par rapport à la } \\
\text { situation actuelle }\end{array}$ \\
\hline
\end{tabular}




\begin{tabular}{|l|l|l|}
\hline Situation actuelle & 1326000 & $0 \%$ \\
\hline Report modal & 1038000 & $-22 \%$ \\
\hline Report modal + covoiturage & 812000 & $-39 \%$ \\
\hline $\begin{array}{l}\text { Report modal + covoiturage }+ \\
\text { technologie }\end{array}$ & 316000 & $-76 \%$ \\
\hline
\end{tabular}

SOURCE : ÉNERgIES DEMAIN, 2007

\subsubsection{Les voies vers le facteur 4 , des choix de société} "l'effet rebond» déjà discuté plus haut: les usagers pourraient compenser les gains d'efficacité énergétique des véhicules par une augmentation des distances parcourues. La diminution de l'intensité énergétique peut avoir pour conséquence une diminution des prix du service rendu au consommateur final. Dès lors, plusieurs effets peuvent être observés : un effet de substitution qui consiste à consommer plus qu'initialement, qui peut se combiner à un effet revenu qui se traduit par la consommation d'autres produits ou services, eux aussi souvent générateurs de GES (Flipo, 2008). Par surcroît, le temps gagné grâce aux technologies de vitesse permet de voyager plus loin, au moins dans la limite des distances "acceptables" (Schneider, 2003). Dans les pays où le prix du carburant est élevé, comme en Europe, l'effet de rebond pourrait atteindre $40 \%$; dans les pays où le prix du carburant est bas, comme aux États-Unis, l'effet de rebond ne devrait pas dépasser $20 \%$. Si l'on tient compte 
des effets de rebond, on constate que les mesures technologiques peuvent réduire les émissions de GES de $5 \%$ à $15 \%$ d'ici 2010 et de $15 \%$ à $35 \%$ d'ici 2020, par rapport à un scénario de base de croissance continue (GIEC, 2001). Si l'amélioration technologique est un levier essentiel (notamment pour les transports en commun ou pour les populations ne disposant pas d'alternatives à la voiture), elle ne peut constituer une voie unique pour atteindre le facteur 4 .

L'étude examine également la portée d'une intervention sur l'aménagement du territoire. En partant des besoins de mobilité, le levier urbanistique consiste à intervenir sur les structures porteuses d'activités économiques et sociales. Il présente ainsi des effets multiples, notamment en influençant les comportements voire les modes de vie en plus de diminuer les distances à parcourir. Il s'agit par exemple de favoriser la mixité fonctionnelle des territoires pour mieux distribuer l'emploi et les services et ainsi diminuer les distances parcourues ou encore de favoriser un partage de la voirie favorable aux transports collectifs et aux modes doux.

Néanmoins, agir sur l'aménagement du territoire implique une intervention structurelle pour modifier la physionomie des territoires. Cela pose de nombreuses questions : en matière de gouvernance territoriale ; pour le rôle des collectivités territoriales et de l'État dans la planification territoriale; en termes de capacités d'investissement publiques et par conséquent de la répercussion des coûts sur les ménages. Ce levier comporte une complexité et des rigidités propres: temporalités longues, investissements massifs, coordination d'acteurs multiples, etc. Mais il génère des "mouvements de fond» en favorisant l'émergence de nouvelles représentations voire de nouvelles valeurs, comme la fabrication de nouvelles proximités par l'usage des modes doux par exemple. De plus, les changements dans l'urbanisme nécessitent aussi un processus démocratique plus participatif, pour un résultat incertain au final.

\subsubsection{Quelles évolutions des structures territoriales?}

51 Se pose alors la question des formes urbaines adaptées aux contraintes énergétiques posées par le facteur 4 : elles remettent en cause la privatisation de l'espace et le phénomène de l'étalement urbain. L'habitat dispersé et la maison pavillonnaire sont l'antithèse de la ville compacte moins consommatrice d'énergie. En effet, les zones peu denses se caractérisent le plus souvent par une moindre accessibilité des équipements (magasins, offre d'emplois suffisamment diversifiée, lieux de récréation, services publics etc.) ainsi qu'une dépendance plus grande à la voiture. Les populations des territoires ruraux et périurbains présentent à cet égard une vulnérabilité importante, notamment lorsque les ménages n'ont pas accès à une offre de transport alternative suffisamment compétitive. Pour ces populations "captives", l'augmentation du coût des énergies entraîne des difficultés en termes d'intégration économique et sociale (accès à l'emploi, aux infrastructures de loisirs, etc.).

52 Le choix de la densification du tissu urbain présente ses propres contraintes dont notamment la saturation des axes de transport, la perte d'aménités (naturelles, paysagères, etc.) et l'accroissement des distances aux espaces naturels. La question des fonctions économiques et sociales des espaces périurbains et ruraux se pose d'autant plus s'ils se situent à proximité de villes compactes.

53 La mise en œuvre du facteur 4 pourrait ainsi créer de nouvelles inégalités - ou tout au moins les révéler - associées aux vulnérabilités de certains territoires, de certaines 
catégories de populations et à des politiques inégalement ciblées. Sous la contrainte des prix du pétrole et du carbone, le non-accès aux fonctions urbaines pourrait par exemple devenir un facteur d'inégalité voire d'exclusion de plus en plus prégnant. De même, se pose la question du niveau de densité socialement acceptable dans les espaces urbains. Ces quelques exemples permettent de dresser un premier panorama des enjeux économiques et sociaux associés à la mise en œuvre du facteur 4 pour les ménages périurbains. Les objectifs ambitieux de réduction des consommations énergétiques et des émissions de gaz à effet de serre imposent de reconsidérer collectivement notre rapport à l'énergie et au territoire.

\section{Limites de l'approche et perspectives pour la recherche et l'action}

55 Les travaux décrits ici n'épuisent pas la question du comment vivre collectivement et individuellement aussi bien et même mieux en émettant quatre fois moins de GES. Cette contrainte sur notre "empreinte carbone » est décrite en premier lieu par l'analyse technique et scientifique, mais il convient de souligner que les choix techniques sont des choix de société éminemment controversés. L'enjeu est ici de donner des points de repère et d'étayer des éléments de débat pour écrire l'histoire du changement économique et social vers le facteur 4 .

Il semble nécessaire de se donner des points d'arrivée (le « facteur 4 ») pour engager une mobilisation et une action. Bien-sûr, cet objectif correspond à un état donné des connaissances et des volontés sur la question du niveau de réponse au changement climatique. Mais le choix politique du facteur 4 suppose de ne pas différer l'action dans un contexte d'incertitude. Une prise en charge sociale et politique est alors d'autant plus importante que les choix techniques ne sont pas neutres en valeur : ces choix engagent ou font l'hypothèse de modifications du vivre ensemble. Par exemple, les mérites énergétiques de la mitoyenneté impliquent le développement de l'habitat collectif et posent des questions en termes de mixité sociale des quartiers, avec de nouvelles contraintes liées à la densité d'habitation (nuisances réelle ou perçues liées au bruit, moins d'espaces verts ou agricoles, etc.).

Les scénarios sont décrits sous une forme technique pour envisager les limites du changement: les temps d'amortissement, les chiffres de construction ou de rénovation décrivent le changement et ses rythmes. Cette analyse est indispensable pour aider à définir un cadre d'intervention pour les acteurs (publics, professionnels, techniciens, etc.). C'est un processus d'exploration pour révéler des obstacles, des problématiques, des facteurs de vulnérabilité, et ainsi contribuer au débat et aider à la prise de décision. Néanmoins, l'analyse technique ne dit pas tout. L'enjeu est de construire une vision intégrée et systémique du facteur 4 , ce à quoi l'étude ne fait que contribuer. Il reste à imaginer les modes de se déplacer, de se loger, de consommer, d'organiser l'action publique, d'aménager les territoires dans une société post carbone.

De ce point de vue, il reste à étudier les transitions entre ce qu'il faudrait faire (selon la rationalité technique et scientifique) et les changements de comportements ou les évolutions des pratiques sociales. Le facteur 4 reste trop souvent décrit comme un horizon-limite peu en phase avec les temps sociaux, sans description des arbitrages voire des choix de société nécessaires. 

redéfinition des conditions du développement et d'approfondissement démocratique. Cette contrainte collective va modifier en profondeur le fonctionnement de nos sociétés. La division des émissions à l'horizon 2050 pose des questions fondamentales notamment en termes de gestion de ressources limitées, de définition et de hiérarchisation des besoins - entre les besoins à satisfaire et les besoins ostentatoires, comme le recommande le Comité Économique et Social Européen (CESE, 2009). On peut donc supposer que le facteur 4 sera producteur de nouvelles contraintes sociales (en matière de mobilité, de localisation résidentielle, de consommation, etc.) mais également producteur de nouvelles normes et représentations positives (par exemple, le recyclage, le troc, l'approvisionnement local, la consommation de produits saisonniers, la circulation en modes doux, etc.).

Des changements sont d'ores et déjà à l'œuvre. Certes, ces comportements restent des "signaux faibles" mais les débats progressent sur les remèdes aux changements climatiques, par exemple au niveau local dans le cadre des plans climat-énergie territoriaux. Dans ces dispositifs sont déployés des efforts d'explication rationnelle des phénomènes observés, et leur transformation en enjeux de politique locale. Ils contribuent à une certaine socialisation des connaissances, et forment une arène où les décideurs, les usagers, les consommateurs, etc. sont interpellés. Ce niveau local permet ainsi l'appropriation démocratique des objectifs du facteur 4. Les objectifs généraux peuvent y devenir des engagements concrets d'actions. Les individus sont d'autant plus disposés à agir que la menace est visible, proche et que les causes sont bien identifiées (Blanchard, 2008). 
64 ne se décrètera pas. Il chemine via l'émergence de valeurs nouvelles : l'autonomie, la valorisation de l'immatériel, l'utilisation optimale des ressources, la valorisation des échanges de proximité (cohérence en termes de lieux de vie), la déconnection de la croissance économique et du bien-être (Dawon, 2010 : 186). Des expériences-pilotes (écoquartier par exemple) érodent progressivement la domination dans les esprits de l'automobile et du pavillon. Loin d'être des invariants, ces constructions sociales qui renvoient à des systèmes de normes, de valeurs et de représentations évoluent dans le temps.

L'amorce de quantification des impacts des scénarios, réalisée pour une région, participe de ces débats. L'impact probable du coût de l'énergie sur le budget des ménages à l'avenir, quelle que soit la politique choisie, nous force à penser le changement. La contrainte climatique et les politiques pour y faire face jouent un rôle majeur dans la remise en cause de nos valeurs et la définition de nouvelles. En étudiant ces changements sociaux induits par la contrainte carbone, on pourra imaginer une transition réussie vers une société post-carbone.

\section{BIBLIOGRAPHIE}

ADEME, 2008, « Comment mettre en place un Fonds Social d'Aide aux travaux de maîtrise de l'énergie en faveur des ménages défavorisés », Guide ADEME, avril

ADEME, 2010, «Les effets rebond des mesures d'efficacité énergétique : comment les atténuer », Lettre Stratégies et études, ${ }^{\circ} 24$, mai

AIE, 2009, World Energy Outlook, Agence Internationale de l'Énergie, Paris

Blanchard O., 2008, «Des attitudes aux comportements : une recherche interdisciplinaire en sciences sociales », Actes du colloque international d'Albi, 25-27 juin, Grenoble, LEPII

Boardman B., Darby S., Killip G., Hinnells M., Jardine C.N., Palmer J. and Sinden G., 2005, 40\% House, Oxford, Environmental Change Unit, University of Oxford, http://www.eci.ox.ac.uk/ research/energy/downloads/40house/40house.pdf

Brown L. R., 2008, Plan B 3.0, Mobilizing to save civilization, Editions Norton, New York.

Cannavò P. F., 2008, « In the Wake of Katrina: Climate Change and the coming crisis of displacement », in S. Vanderheiden (ed.), Political Theory and Global Climate Change, MIT Press, Cambridge.

Cérézuelle D., 2007, « Crise du "savoir habiter", exclusion sociale et accompagnement à l'autoréhabilitation du logement », IDDRI, Idées pour le débat $n^{\circ} 5$, http://www.iddri.org/ Publications/Collections/Idees-pour-le-debat/id_0705_cerezuelle_svrfairelocx.pdf

CESE, 2009, Avis du Comité économique et social européen sur « Les conséquences sociales de l'évolution du binôme transports/énergie ", Bruxelles 2009/C 175/08, http://eur-lex.europa.eu/ LexUriServ/LexUriServ.do ?uri =OJ :C :2009: 175:0043:0049 :FR :PDF 
Criqui P., Mima S., Rynikiewicz C., 2006, « Prospective énergétique à 2050, contrainte carbone et changements structurels », LEPII EPE, http://webu2.upmf-grenoble.fr/iepe/textes/PC-SMCR_FONDDRIavril_260406.pdf

Dawon J., 2010, "Ecovillages and the transformation of values", in Worldwatch Institute, State of the world - Transforming cultures, p. 185-190, http://www.gaiaeducation.org/docs/Ecovillages\% 20Transformation\%20Values.pdf

De Boissieu C. (éd.), 2006, Rapport du Groupe de travail « Division par quatre des émissions de gaz à effet de serre de la France à l'horizon 2050 », La documentation française, Paris.

Deffeyes K.S. 2001, Hubbert's Peak, the impending world oil shortage, Princeton university Press, Princeton États-Unis.

Flipo F., Gossart C., 2008, « Infrastructure numérique et environnement : l'impossible domestication de l'effet rebond ", Colloque international Services, innovation et développement durable, Poitiers, 26-28 mars.

German Advisory Council on Climate Change, 2008, Climate Change as a Security Risk, Earthscan, Londres, Berlin

GIEC, 2001, Troisième rapport du GIEC, résumé technique du Groupe de Travail III. http:// www.grida.no/publications/other/ipcc_tar/ ?src =/climate/IPCC_tar/vol4/french/174.htm

Hansen J., Sato M., Kharecha P., Beerling D., Masson-Delmotte V., Pagani M., Raymo M., Royer D. L., Zachos J. C., 2008, « Target Atmospheric CO2: Where Should Humanity Aim?”, Goddard Institute for Space Studies, NASA, New York, http://www.columbia.edu/ jeh1/2008/ TargetCO2_20080407.pdf

Héran F., 2006, « De la dépendance automobile », Ecorev' - Revue critique d'écologie politique, $\mathrm{n}^{\circ} 24$, http://ecorev.org/spip.php ?article542

IEEP, 2009, " Action taken to address climate and energy issues across EU Member States, and prospects for implementation of the climate action and renewable energy package - A Review for the European Climate Foundation Bruxelles", juin

INSEE 1996, « Le logement - reconstruction, grands ensembles et accession à la propriété », Paris, INSEE Première $\mathrm{n}^{\circ} 456$

Kasparian J., 2008, « Contribution of crude oil price to households' budget: the weight of indirect energy use », Energy Policy, 37 (1), p. 111-114

Latour B., 1999, Politiques de la nature. Comment faire entrer les sciences en démocratie?, La Découverte, Paris

Lecœur E., 2008 « Le changement d'attitude face à la crise climatique : les enjeux sociologiques et psychosociologiques », Les cahiers de l'IAURIF, n 147, p. 230-244

PNUD, 2007, "Fighting climate change: Human solidarity in a divided world", Human Development Report 2006-2007 (HDR)

Radanne P., 2004, «La division par 4 des émissions de dioxyde de carbone en France d'ici à 2050 ", Rapport à la Mission Interministérielle à l'Effet de Serre (MIES), Paris

Radanne P., 2005, « Facteur Quatre et aménagement du territoire », Territoires 2030, Datar, n² , p. 63-70

Saglio P., Chosson A. 2010, « Transformation des modes de vie, des comportements et de la consommation », Paris, Rapport au Ministre d'État, ATD-Quart-Monde et CSCV, février, http:// lesrapports.ladocumentationfrancaise.fr/BRP/104000092/0000.pdf 
Schneider F., 2003, « L'effet rebond », L'Ecologiste, $\mathrm{n}^{\circ}$ 11, octobre, p. 45

Stern N., 2006, « Stern Review, the economics of Climate Change », DTI Londres

Traisnel J.-P. et al., 2001, « Habitat et développement durable », Les cahiers du CLIP nº 13

Traisnel J.-P., Raux C., 2007, « Habitat et déplacement dans les aires urbaines », Les annales de la recherche urbaine, $\mathrm{n}^{\circ} 103$

Virage Énergie Nord Pas-de-Calais, 2008, «Énergies d'avenir en Nord-Pas-de-Calais - Quelles solutions au dérèglement climatique? ", janvier

\section{NOTES}

1. La loi de programme sur les orientations de la politique énergétique (LOE) du 13 juillet 2005 engage la France sur une diminution de $3 \%$ par an des émissions de GES.

2. Les fourchettes de valeurs expriment des pourcentages; le chiffre entre parenthèses correspond au nombre de communes concernées pour chaque fourchette.

3. Revenus fiscaux des ménages 2004, INSEE 2007.

4. Source INSEE, recensements 1999 et 2006.

5. Les objectifs du facteur 4 correspondent à un niveau de réduction des émissions de GES liées au chauffage de l'ordre de $85 \%$. Dans ce scenario, on réhabilite pour cela $55 \%$ du parc actuellement construit de manière lourde (ce qui présente un potentiel de diminution des consommations d'énergie totales du logement de l'ordre de $75 \%$ ).

6. Distance parcourue annuellement par ménage tous motifs confondus : travail, scolaire, achats, loisirs, motifs personnels (démarches administratives, visites familiales, etc.), motifs professionnels (hors domicile-travail).

\section{RÉSUMÉS}

L'objectif de division par quatre des émissions de gaz à effet de serre à l'horizon 2050 aura des conséquences majeures sur les territoires, les ménages, les entreprises. Si l'objectif est global, les conséquences des changements drastiques nécessaires pour y parvenir seront localisées. Nous avons étudié les conséquences de la réduction forte des émissions de gaz à effet de serre dans la région Nord-Pas-de-Calais à l'horizon 2020-2050, notamment pour la réhabilitation du logement et le transport des personnes. Une quantification des conséquences budgétaires de ces politiques incontournables montre que la contrainte devient forte voire insoutenable pour la majorité des ménages. Ces résultats conduisent à dresser une déclinaison des enjeux et des politiques sur le territoire régional.

Cutting the greenhouse gas emissions by four by 2050 will have major consequences on local economies, households and policies. This objective is global, however, consequences will have to be faced and dealt with locally. We have studied the consequences of deep reductions of greenhouse gas emissions in the Nord Pas-de-Calais region by 2020-2050, in particular regarding housing renovation and mobility. Modelling the financial consequences of these necessary 
policies show that constraints become strong and even unbearable for a majority of household. These results call for well-informed and locally-based policies.

\section{INDEX}

Keywords : mitigation policies, greenhouse gas emissions, modelling, household budget, social consequences

Mots-clés : réductions d'émissions de gaz à effet de serre, modélisation, budget des ménages, conséquences sociales

\section{AUTEURS}

\section{ANNE-SOPHIE STEVANCE}

Anne-Sophie Stevance est diplômée de l'Institut d'Études Politiques de Lille, spécialisation en politiques territoriales de développement durable, recherche sur la commande publique et le développement durable. Chargée d'études environnement et énergie chez Énergies Demain. Spécialisation dans l'accompagnement des collectivités pour la définition de stratégies énergieclimat (plans climat-énergie territoriaux). annesophie.stevance@energies-demain.com 16bis rue François Arago 93100 Montreuil sous Bois Tél. 01.42.87.23.27

\section{NICOLAS HOUDANT}

Nicolas Houdant est directeur d'Énergies Demain. Spécialisé dans le conseil stratégique aux collectivités en matière de maîtrise de l'énergie et de réduction des émissions de gaz à effet de serre ainsi que dans la modélisation des consommations d'énergie dans le logement, le transport, l'électricité. Concepteur de méthodes et d'outils d'abord à l'INESTENE (Institut d'évaluation des stratégies énergétiques européennes) puis chez Énergies Demain. Par ailleurs enseignant à l'École des Mines de Paris (cycle de vie des systèmes énergétiques). nicolashoudant@energiesdemain.com 16 bis rue François Arago 93100 Montreuil sous Bois Tél. 01.42.87.23.27

\section{ANTOINE BONDUELLE}

Antoine Bonduelle est gérant du bureau d'études E\&E Consultant. Pratique la veille technologique comme journaliste scientifique puis consultant depuis 1983 dans les champs de l'environnement et de l'énergie. Il a aussi été responsable des ONG françaises aux conférences sur le changement climatique à partir de celle de Berlin (1995) et a co-fondé le Réseau Action ClimatFrance (RAC-F). Il est ingénieur ICAM, diplômé en journalisme (Université Laval, Québec) et titulaire d'un DEA de Sciences Politiques (Lille II). antoine.bonduelle@ee-consultant.fr 900 vieux chemin de Saint-Omer 59670 Cassel Tel. 03.66.72.10.05 